Utah State University

DigitalCommons@USU

$1-1-2013$

\title{
OSIRIS-Rex OCAMS Detector Assembly Characterization
}

J. Hancock

B. Crowther

M. Whiteley

R. Burt

M. Watson

J. Nelson

See next page for additional authors

Follow this and additional works at: https://digitalcommons.usu.edu/sdl_pubs

\section{Recommended Citation}

Hancock, J.; Crowther, B.; Whiteley, M.; Burt, R.; Watson, M.; Nelson, J.; Fellows, C.; Rizk, B.; Kinney-Spano, E.; Perry, M.; and Hunten, M., "OSIRIS-Rex OCAMS Detector Assembly Characterization" (2013). Space Dynamics Lab Publications. Paper 55.

https://digitalcommons.usu.edu/sdl_pubs/55

This Article is brought to you for free and open access by the Space Dynamics Lab at DigitalCommons@USU. It has been accepted for inclusion in Space Dynamics Lab Publications by an authorized administrator of DigitalCommons@USU. For more information, please contact digitalcommons@usu.edu.

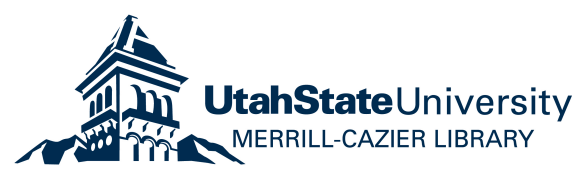




\section{Authors}

J. Hancock, B. Crowther, M. Whiteley, R. Burt, M. Watson, J. Nelson, C. Fellows, B. Rizk, E. Kinney-Spano, M. Perry, and M. Hunten 


\title{
OSIRIS-REx OCAMS detector assembly characterization
}

\author{
J. Hancock*a , B. Crowther ${ }^{\mathrm{a}}$, M. Whiteley, , R. Burt ${ }^{\mathrm{a}}$, M. Watson ${ }^{\mathrm{a}}$, J. Nelson $^{\mathrm{a}}$, C. Fellows ${ }^{\mathrm{b}}$, B. Rizk ${ }^{\mathrm{b}}$, \\ E. Kinney-Spano ${ }^{\mathrm{b}}$, M. Perry ${ }^{\mathrm{b}}$, M. Hunten ${ }^{\mathrm{b}}$ \\ ${ }^{a}$ Space Dynamics Laboratory, Utah State University Research Foundation, 1695 N. Research Park \\ Way, N. Logan, UT, USA 84341; 'bunar Planetary Lab, University of Arizona, 1415 N. Sixth \\ Avenue, Tucson, AZ, USA 85705
}

\begin{abstract}
The OSIRIS-REx asteroid sample return mission carries a suite of three cameras referred to as OCAMS. The Space Dynamics Laboratory (SDL) at Utah State University is providing the CCD-based detector assemblies for OCAMS to the Lunar Planetary Lab (LPL) at the University of Arizona. Working with the LPL, SDL has designed the electronics to operate a $1 \mathrm{~K}$ by $1 \mathrm{~K}$ frame transfer Teledyne DALSA Multi-Pinned Phase (MPP) CCD. The detector assembly electronics provides the CCD clocking, biasing, and digital interface with the OCAMS payload Command Control Module (CCM). A prototype system was built to verify the functionality of the detector assembly design and to characterize the detector system performance at the intended operating temperatures. The characterization results are described in this paper.
\end{abstract}

Keywords: OSIRIS-REx, OCAMS, detector as sembly, CCD characterization

\section{INTRODUCTION}

The Lunar Planetary Laboratory (LPL) at the University of Arizona is providing the OSIRIS-REx Camera Suite (OCAMS) for the OSIRIS-REx sample and return mission to the asteroid Bennu. The OCAMS payload consists of three visible cameras, the PolyCam, MapCam, and SamCam. The PolyCam is an eight inch telescope used to first acquire the asteroid at long ranges and provide high resolution imagery at close ranges. The MapCam will map the asteroid in four different colors and provide context imagery of the sample site and the SamCam continuously documents the sample acquisitions. The three cameras work together to provide global mapping, sample site reconnaissance and characterization, high resolution imaging, and record the sample acquisition ${ }^{1}$.

All three OCAMS cameras are designed to use identical detector assemblies provided by the Space Dynamics Lab (SDL) at Utah State University. SDL is responsible for the design, fabrication, environmental testing, and electro-optical characterization of the OCAMS detector assemblies. This paper will discuss the results of the electro-optical characterization of the first engineering qualification unit fabricated by SDL.

\section{DETECTOR ASSEMBLY DESIGN}

The OCAMS detector assembly consists of electrical, mechanical, and thermal hardware designed to a common interface, allowing the assembly to be used interchangeably in any of the three OCAMS cameras. The camera systems use a front illuminated CCD as their integrated sensors. The OCAMS CCD is a $1 \mathrm{k}$ by $1 \mathrm{k}$ frame transfer CCD with 8.5 micron pixels manufactured by Teledyne DALSA. The CCD has two output taps that vertically separate the readout of the image storage area. The detector assembly is designed to operate the CCD in single or dual-tap mode. Upon receiving each CCD, SDL performs physical inspection of the device by digital microscopy and an initial characterization verifying CCD performance before the CCD is installed in the detector assembly. A model of the detector assembly is shown in Figure 1.

*jed.hancock@sdl.usu.edu; phone 1435 713-3523; fax 1435 713-3501; www.spacedynamics.org

UV/Optical/IR Space Telescopes and Instruments: Innovative Technologies and Concepts VI,

edited by Howard A. MacEwen, James B. Breckinridge, Proc. of SPIE Vol. 8860, 88600J

(C) 2013 SPIE · CCC code: $0277-786 X / 13 / \$ 18 \cdot$ doi: $10.1117 / 12.2024665$ 


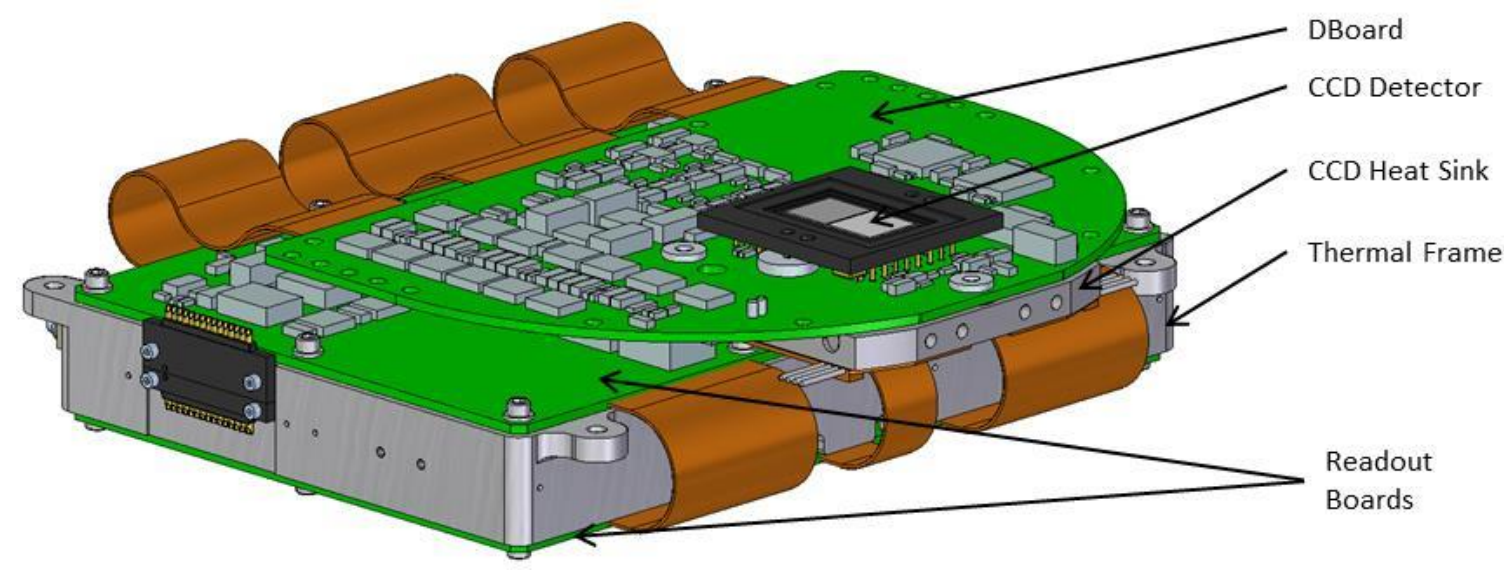

Figure 1. The OCAMS detector assembly.

The active area of the CCD is precision-aligned during installation of the CCD package to the printed circuit board (PCB). As illustrated above, a heat sink is attached to the CCD and regulates the operation temperature.

The detector assembly electronics interface to the CCM which provides power and issues commands. The detector as sembly electronics are fabricated using a three-section rigid-flex PCB. The DBoard houses the CCD and biasing and clocking electronics. The middle readout board contains the circuitry to perform voltage regulation for the various clock levels required by the CCD. Also found on the middle readout board is the analog signal conditioning leading up the analog-to-digital converter. The bottom readout board contains the FPGA and interface electronics. The FPGA provides clocking to the CCD, stores images in memory, and controls the data interface to the CCM.

The PCBs are mounted on a thermal frame that provides both the mechanical and thermal interface to the OCAMS cameras. Temperature sensors and heaters are installed in strategic locations to provide feedback and control to the temperature control system in the CCM.

\section{CHARACTERIZATION PLAN}

SDL has developed an assembly, integration, and test plan to ensure that the detector assemblies are fabricated using program approved materials, processes, and procedures. The performance of the detector array is checked at various stages throughout the build process, once the assembly has passed vibration and thermal vacuum testing, a final characterization is performed to verify the as-delivered performance of the detector assembly subsystems to the cameras.

\subsection{Characterization matrix}

The final characterization of the detector assembly consists of data collection and data analysis. The detector assembly test plan is designed to collect data that can be analyzed to establish the performance metrics of the detector assembly and ensure compliance to key performance requirements. Table 1 is the test matrix produced for the final characterization testing of the assembly. The table lists the performance metric that will be characterized, the test procedure that will be followed to generate the data to compute the performance metric, the source configuration, and the temperature ranges the metric is characterized over.

Table 1. The characterization matrix for the OCAMS detector assemblies.

\begin{tabular}{|l|l|l|l|}
\hline \multicolumn{1}{|c|}{ Performance Metric } & \multicolumn{1}{|c|}{$\begin{array}{c}\text { Test Procedure } \\
\text { (Data Collection) }\end{array}$} & \multicolumn{1}{c|}{$\begin{array}{c}\text { Source } \\
\text { Configuration }\end{array}$} & $\begin{array}{c}\text { Temperature } \\
\text { Range }\end{array}$ \\
\hline Dark Current & Dark Response & Dark & -30 to $40^{\circ} \mathrm{C}$ \\
\hline Read Noise & Dark Response & Dark & -30 to $40^{\circ} \mathrm{C}$ \\
\hline Gain & PTC & LED & -30 to $40^{\circ} \mathrm{C}$ \\
\hline Pixel Gain Nonlinearity & PTC & LED & -30 to $40^{\circ} \mathrm{C}$ \\
\hline Dynamic Range & PTC & LED & -30 to $40^{\circ} \mathrm{C}$ \\
\hline
\end{tabular}




\begin{tabular}{|l|l|l|l|}
\hline \multicolumn{1}{|c|}{ Performance Metric } & \multicolumn{1}{|c|}{$\begin{array}{c}\text { Test Procedure } \\
\text { (Data Collection) }\end{array}$} & $\begin{array}{c}\text { Source } \\
\text { Configuration }\end{array}$ & $\begin{array}{c}\text { Temperature } \\
\text { Range }\end{array}$ \\
\hline Photon Response Non-Uniformity & HCTE & LED & -30 to $40^{\circ} \mathrm{C}$ \\
\hline VCTE & VCTE & LED & -30 to $40^{\circ} \mathrm{C}$ \\
\hline HCTE & HCTE & LED & -30 to $40^{\circ} \mathrm{C}$ \\
\hline
\end{tabular}

Four data collection events are performed to characterize the performance metrics for this sensor: dark response, photon transfer curve (PTC), horizontal charge transfer efficiency (HCTE), and vertical charge transfer efficiency (VCTE). Anti-blooming performance testing is performed on CCDs manufactured with a specialized mask. The anti-blooming characterization is not reported here.

\section{Dark Response}

The dark response data collection captures images that are processed to measure the offset, dark current, and read noise of the detector electronics assembly. Dark response data is collected with the source turned off to provide a dark background for the detector array. Dark images will be captured at varying integration times and temperatures. Read noise data will only be collected as a function of temperature.

\section{Photon Transfer Curve (PTC)}

The photon transfer curve data characterizes the temporal noise observed for a range of integrated signal levels. Image data is collected at multiple points in the dynamic range from the smallest exposure time $(0$ seconds) to the largest exposure time (full well). CCD signal and noise data are collected using the LED source; dark frames are also collected at each exposure level for background subtraction. Data will be collected at varying CCD exposure times and temperatures.

The performance metrics obtained through the PTC testing include gain (e-/DN), pixel gain nonlinearity, and dynamic range.

\section{HCTE}

The horizontal charge transfer efficiency data collection measures the per-pixel charge transfer efficiency of the horizontal registers. To collect the data, the horizontal registers are over-scanned for each row of the CCD. The data is collected at approximately $1 / 2$ well fill and over various temperatures. The HCTE collection also provides the data to compute the photon response non-uniformity (PRNU).

\section{VCTE}

The vertical charge transfer efficiency data collection and analysis measures the per-pixel charge transfer efficiency of the CCD in the vertical transfer direction. The VCTE data is also collected using the over-scan technique. VCTE data is collected for each column in the CCD by over-clocking in the vertical direction. The data is collected at approximately $1 / 2$ well fill and over various temperatures.

\subsection{Test equipment}

SDL operates the detector assemblies using a custom ground station that simulates the electrical functionality of the CCM by providing power, command, and data interfaces. The ground station is built using commercially available computer hardware, custom cabling, and custom developed software for camera commanding and image display.

The final characterization of the OCAMS detector assembly occurs in a temperature controlled thermal vacuum (TVAC) chamber that has been modified to provide a flight simulated environment of the electrical, thermal, and mechanical interfaces. Figure 2 shows the TVAC chamber opened with a detector assembly installed. 


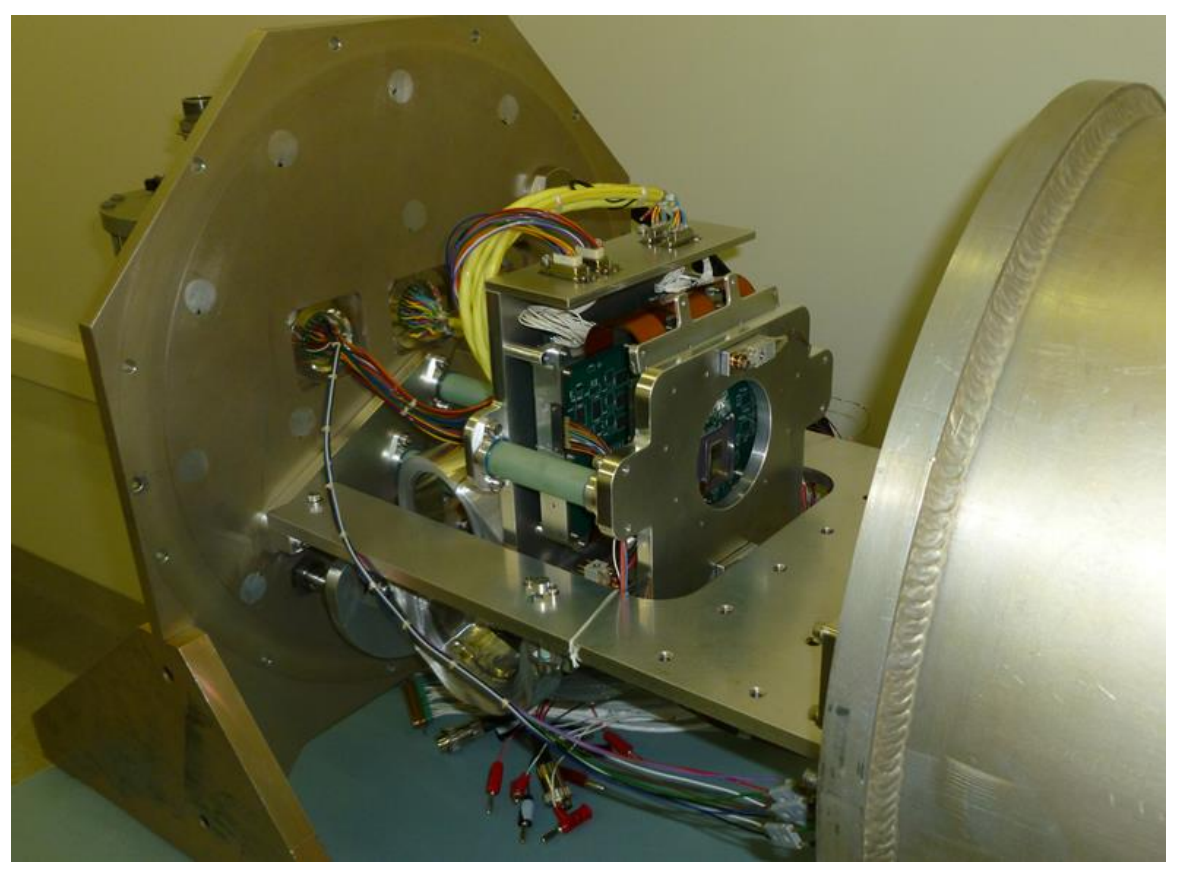

Figure 2. The OCAMS detector assembly TVAC test station.

The TVAC chamber uses a two-stage cryocooler, flexible thermal links, temperature sensors, and three zone heaters to control the detector assembly interfaces to the prescribed test temperatures. The OCAMS detector assembly is mounted onto two separate brackets (zones), each cooled by a thermal link attached to the helium cold-head stage and then heated by a temperature controller for set point temperature control. In addition, the CCD heat sink is controlled independently via a thermal link and the third zone heater. Temperature sensors mounted on the test and flight hardware are used for monitoring and control during the final characterization.

The detector assembly is mounted in the test chamber in close proximity to an optical source made of LEDs and diffusing elements. The optical source is mounted in the chamber directly in front of the detector array providing a uniform source of illumination over the CCD array.

\section{CHARACTERIZATION RESULTS}

The final characterization of the first engineering qualification detector assembly occurred at SDL in July of 2013. Data was collected for $\mathrm{CCD}$ temperatures ranging from $-30^{\circ} \mathrm{C}$ to $+40^{\circ} \mathrm{C}$. The preliminary data analysis has computed performance metrics for the CCD when operated at approximately $23^{\circ} \mathrm{C}$. The results are presented in the following sections.

\subsection{Dark current and read noise}

The read noise of the detector was calculated by computing the standard deviation of the pixel response from several frames with an integration time setting of 0 seconds. Because the CCD is a frame transfer device, the actual minimum integration time is approximately $2 \mathrm{~ms}$. The read noise of the detector assembly was calculated for each tap, the left tap read noise performance is 37 electrons and the right tap read noise performance is 34 electrons.

The dark current was estimated by computing mean pixel response from several frames at 5 different integration times. Figure 3 is a plot of the pixel response from the left tap of the device for integration times ranging from 0 to $448 \mathrm{~ms}$. A linear fit is applied to the data and the slope of the fit is an estimate of the dark current of the device. The dark current at approximately $23^{\circ} \mathrm{C}$ was computed to be $85 \mathrm{e}-/ \mathrm{pix} / \mathrm{sec}$. 


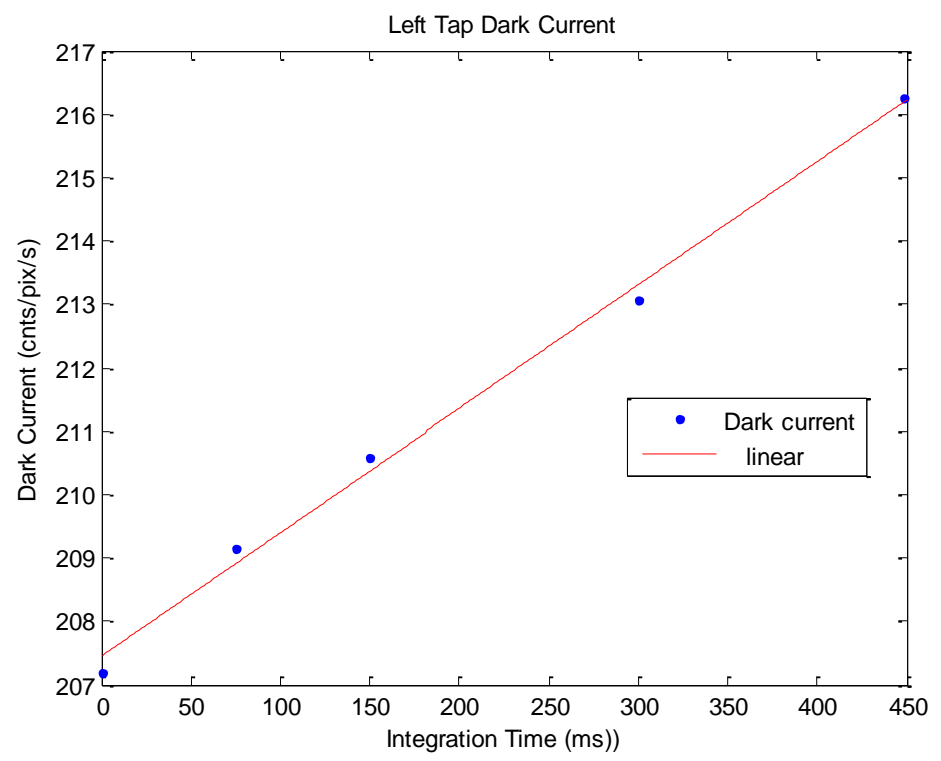

Figure 3. The CCD dark current performance of the left output tap.

\subsection{Gain, gain non-linearity, and dynamic range}

The photon transfer measurement characterizes the temporal noise observed for a range of integrated signal levels. The range of signal levels cover the dynamic range of the sensor and is incremented by adjusting the camera exposure from the shortest possible value until the detector is saturated. A photon transfer curve is created by plotting the mean pixel response versus the noise throughout the dynamic range of the device. Figure 4 is the photon transfer curve of the right tap of the CCD on a log scale plot. The three regions of the CCD response are recognized on the plot as the read noise, shot noise, and saturated regions ${ }^{2}$.

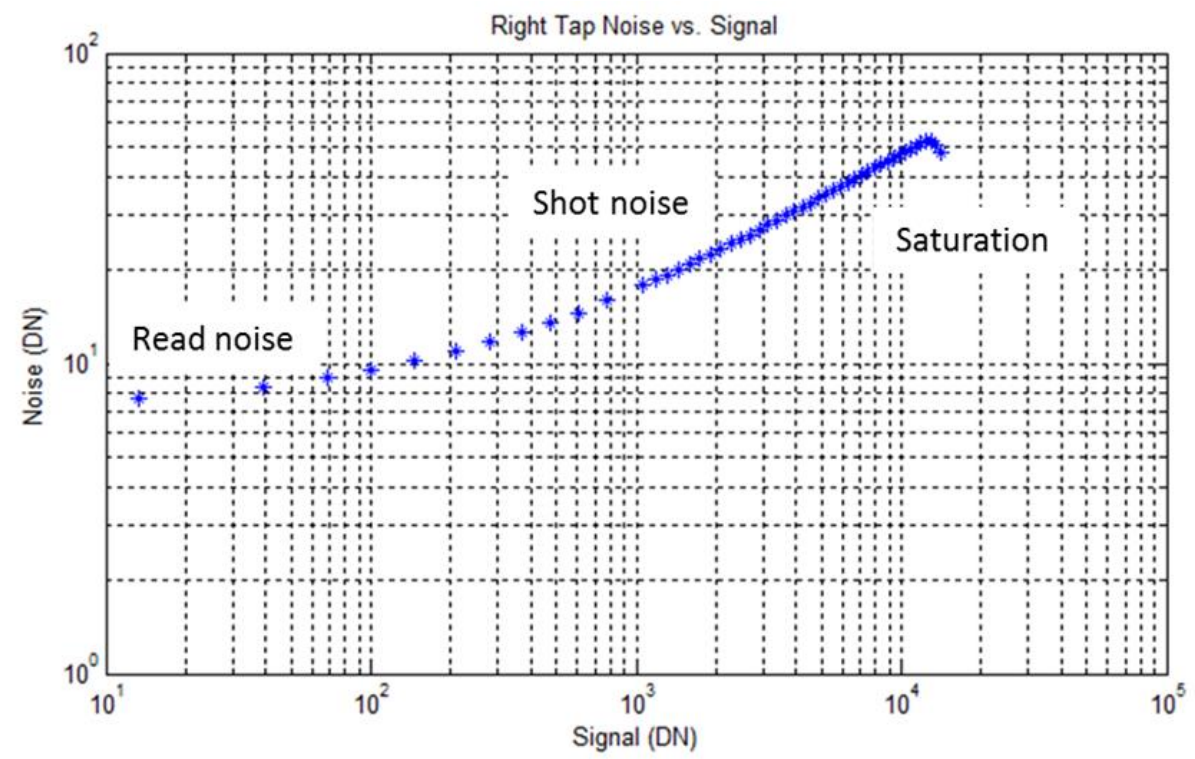

Figure 4. Photon transfer curve plotted on a log scale for the right output tap.

The gain, gain non-linearity, and full well of the detector assembly are all computed using photon statistics. The gain, in units of e-/DN, is defined as the conversion between the number of electrons in the CCD pixel to the number of digital counts recorded. The gain is obtained from the shot noise dominated portion of the photon transfer curve. This portion of the curve is dominated by shot noise, in that the noise is proportional to the square root of the number of electrons. The 
CCD gain is then calculated as the signal divided by the variance at each individual point on the linear portion of the photon transfer curve. The average gain is calculated to be $4.38 \mathrm{e}-/ \mathrm{DN}$ and $4.42 \mathrm{e}-/ \mathrm{DN}$ for the left and right tap respectively.

Figure 5 is a plot of the variance versus signal for the right output tap of the CCD and highlights the pixel well depth. The pixel well depth, or full well, is determined when the variance (or noise) begins to decrease, this is the region of pixel satuation. The dynamic range will be defined as the range of signal levels between $10 \%$ and $90 \%$ of the pixel well depth divided by the read noise. The dynamic range of right output tap was found to be 1216 , and the left was calculated as 1326 .

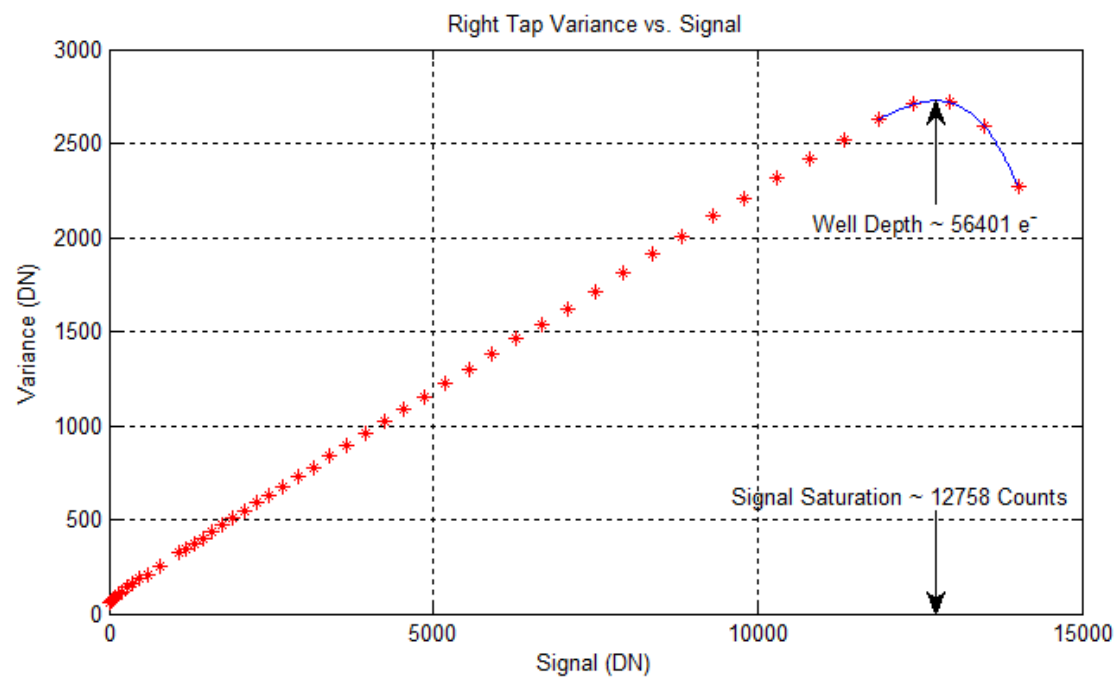

Figure 5. The variance plotted versus signal for the right output tap.

The pixel gain non-linearity of the CCD right output tap is plotted in

Figure 6. In the figure, the gain is plotted versus signal acros s the dynamic range. The non-linearity is computed as the error of the rms residuals to a best fit linearity correction factor. The pixel gain non-linearity of the left tap is $1.03 \%$ and the non-linearity of the right tap is $0.95 \%$. As shown in Figure 6, the gain of the detector assembly increases with signal level.

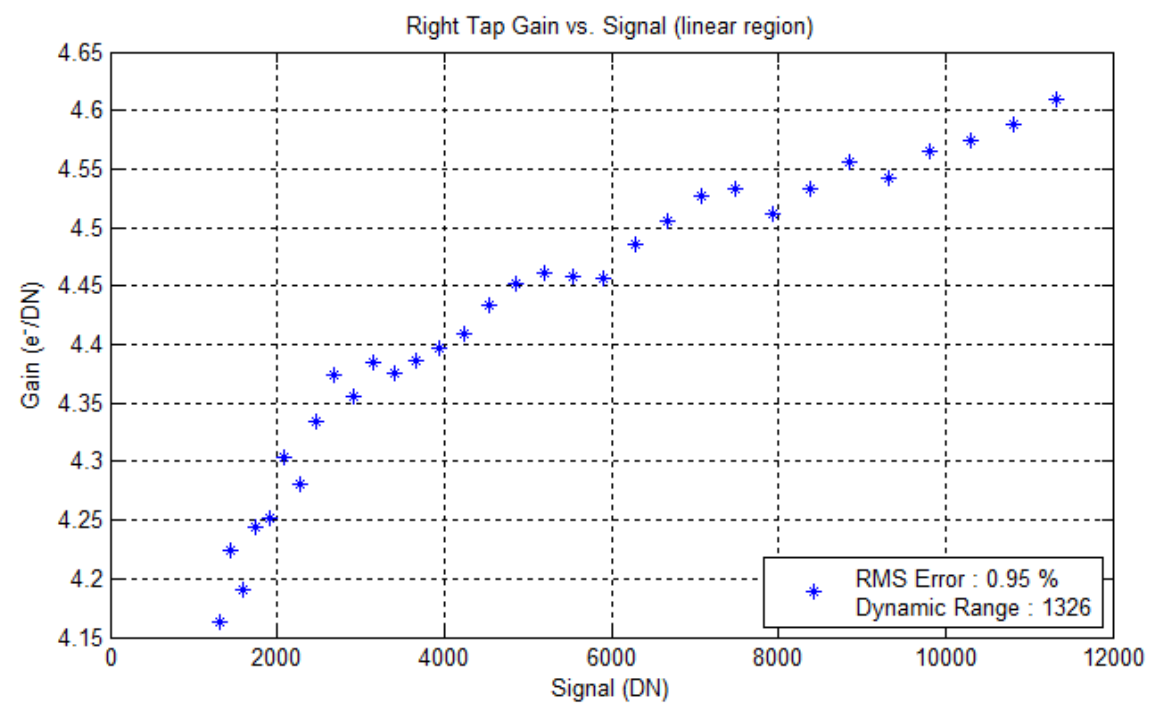

Figure 6. Pixel gain non-linearity of the CCD right output tap. 


\subsection{Photon response non-uniformity}

The PRNU quantifies the fixed pattern noise of the detector assembly. To calculate the PRNU of the system, several frames are collected with the same exposure at approximately half well fill and under uniform illumination. The PRNU is computed by calculating the pixel by pixel average of several frames, and then computing the spatial standard deviation of the averaged frame. The PRNU of the left tap, defined as the spatial standard deviation at half well fill divided by the full well is $0.67 \%$. The PRNU of the right tap is $0.89 \%$. It is also common to define the PRNU at half well fill as the spatial standard deviation divided by the mean pixel value. Defined in this manner, the PRNU values are $1.40 \%$ and $1.82 \%$ for the left and right taps.

\subsection{VCTE}

The VCTE for each column in the CCD array is plotted in Figure 7. The VCTE for each column in the CCD array is computed by averaging 30 frames under uniform illumination, and calculating CTE using the over-scan technique. The VCTE of the CCD has a column dependency that is being investigated.

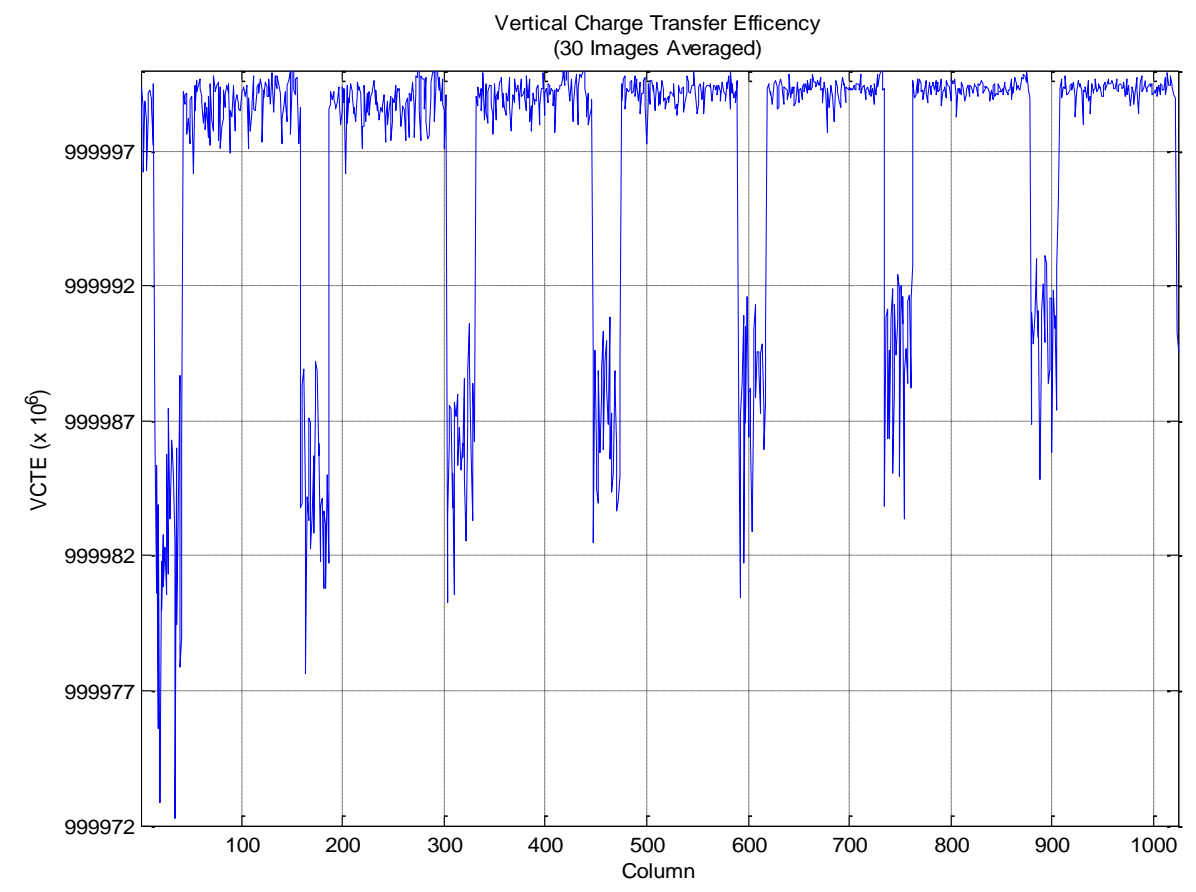

Figure 7. The VCTE for each column of the CCD.

\subsection{HCTE}

The HCTE for each row of the CCD is plotted in Figure 8. The HCTE is also computed by averaging 30 frames and using the over-scan technique. 

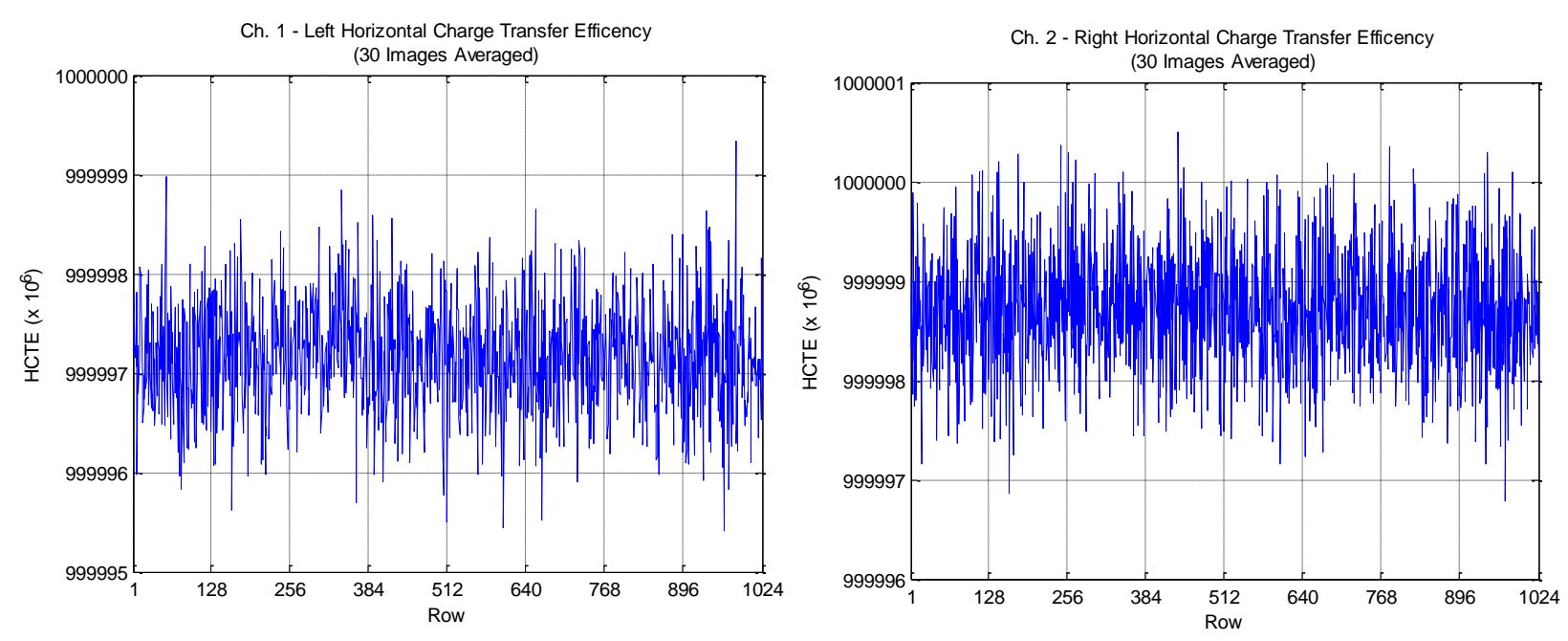

Figure 8. The HCTE of the left and right output taps of the CCD.

\section{SUMMARY}

The first of the OCAMS detector assembly engineering qualification models has been fabricated, environmentally tested, and electro-optically characterized at SDL. The detector assembly performance, operating with a CCD temperature of $20^{\circ} \mathrm{C}$ under TVAC, complies with all of the electro-optical requirements.

Table 2. A summary of the OCAMS detector assembly performance.

\begin{tabular}{|l|l|l|l|}
\hline \multicolumn{1}{|c|}{ Performance Metric } & \multicolumn{1}{c|}{ Requirement } & \multicolumn{1}{c|}{ Left Tap } & \multicolumn{1}{c|}{ Right Tap } \\
\hline Dark Current & $2500 \mathrm{e}-/ \mathrm{pix} / \mathrm{sec}$ & $85 \mathrm{e}-/ \mathrm{pix} / \mathrm{sec}$ & $92 \mathrm{e}-/ \mathrm{pix} / \mathrm{sec}$ \\
\hline Read Noise & $50 \mathrm{e}-$ & $37 \mathrm{e}-$ & $34 \mathrm{e}-$ \\
\hline Gain & Adjustable & $4.38 \mathrm{e}-/ \mathrm{DN}$ & $4.42 \mathrm{e}-/ \mathrm{DN}$ \\
\hline Pixel Gain Nonlinearity & $1.5 \%$ & $1.03 \%$ & $0.95 \%$ \\
\hline Dynamic Range & 600 & 1216 & 1326 \\
\hline Photon Response Non-Uniformity & $5 \%$ & $0.67 \%$ & $0.89 \%$ \\
\hline VCTE & 0.99995 & 0.999972 & 0.999982 \\
\hline HCTE & 0.99995 & 0.999997 & 0.999998 \\
\hline
\end{tabular}

\section{ACKNOWLEDGEMENTS}

We would like to acknowledge each of our partners at NASA, Teledyne Dalsa, LPL, and SDL who have contributed to the development of OCAMS detector assemblies.

\section{REFERENCES}

[1] Lunar Planetary Lab, OSIRIS-REx, (2013), http://osiris-rex.lpl.arizona.edu/

[2] Janesick, J. R., [Scientific charge-coupled devices], Bellingham, Wash.: SPIE Press, (2001). 